\title{
Is there a golden rule for the stochastic Solow growth model?
}

\section{Working Paper}

Author(s):

Schenk-Hoppé, Klaus Reiner

Publication date:

2001

Permanent link:

https://doi.org/10.3929/ethz-a-004373335

Rights / license:

In Copyright - Non-Commercial Use Permitted

Originally published in:

Working paper / Institute for Empirical Research in Economics 33 


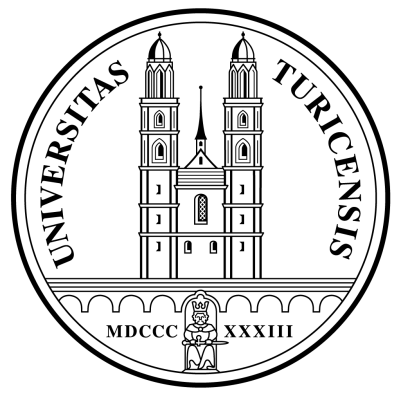

Institute for Empirical Research in Economics

University of Zurich

Working Paper Series

ISSN 1424-0459

forthcoming in Macroeconomic Dynamics

Working Paper No. 33 (Revised version)

Is There A Golden Rule

For The Stochastic Solow Growth Model?

Klaus Reiner Schenk-Hoppé

January 2001 


\title{
Is There A Golden Rule \\ For The Stochastic Solow Growth Model?
}

\author{
Klaus Reiner Schenk-Hoppé * \\ Institute for Empirical Research in Economics \\ University of Zurich, Switzerland
}

\begin{abstract}
Postal address:
Institute for Empirical Research in Economics

University of Zurich

Blümlisalpstrasse 10

CH-8006 Zürich, Switzerland

phone $+41(0) 1-6343714$

fax $+41(0) 1-6344907$

klaus@iew.unizh.ch
\end{abstract}

June 14, 2001

\footnotetext{
${ }^{*}$ Research supported in part by Deutsche Forschungsgemeinschaft while the author was at the Department of Economics, University of Bielefeld. I am grateful to an anonymous referee whose comments have helped to distinctly improve this paper.
} 


\begin{abstract}
This paper analyzes the dependence of average consumption on the saving rate in a one-sector neoclassical Solow growth model with production shocks and stochastic rates of population growth and depreciation where arbitrary ergodic processes are considered. We show that the long-run behavior of the stochastic capital intensity, and hence average consumption along any sample-path, is uniquely determined by a random fixed point which depends continuously on the saving rate. This result enables us to prove the existence of a golden rule saving rate which maximizes average consumption per capita. We also show that the golden rule path is dynamically efficient. The results are illustrated numerically for Cobb-Douglas and CES production function.
\end{abstract}

Key words and phrases. Stochastic Solow model, Golden Rule, random fixed points, random dynamical systems.

JEL classification numbers. E13, C60, O41.

\title{
1 Introduction
}

The existence of a golden rule saving rate for the Solow growth model is a standard textbook problem, see e.g. Phelps (1966) and Barro and Salai-Martin (1995). The analogous question for the stochastic Solow model, however, has not been studied in the literature so far to the author's best knowledge. If productivity, population growth, or depreciation is subject to exogenous perturbations, the analysis of the descriptive Solow growth model requires methods different from those applied in the deterministic case. A first analysis of this stochastic growth model is due to Mirman $(1972,1973)$. Using the framework of Markov processes, he could prove existence and uniqueness of stationary probability measures (Markov equilibria) for the Solow model with i.i.d. (independent and identically distributed) production shocks.

In the last 25 years, our understanding of the dynamical properties of non-linear stochastic dynamical systems has improved dramatically. This opens the door to the study of stochastic economic models and problems that have not been tractable before. In this paper, we apply random dynamical systems theory for the study of the stochastic Solow model. This theory, which is comprehensively presented in the monograph by Arnold 
(1998), provides a coherent mathematical description of non-linear dynamical systems with randomness. An increasing number of methods for the analysis of stochastic systems is being developed using this framework.

Here, we employ the concept of a random fixed point to prove the existence of a golden rule saving rate for the stochastic Solow model in which the productivity and the rates of population growth and depreciation are subject to perturbations stemming from arbitrary ergodic processes. This approach has been used in Schenk-Hoppé and Schmalfuss (in press) to describe the long-run behavior of the capital stock in the stochastic Solow model. We will apply their main result on the existence of a unique globally attracting random fixed point.

Due to the presence of exogenous shocks, consumption in each period depends on the past realization of shocks and the initial capital stock. We will first prove that average consumption along each sample-path of the stochastic Solow growth model is well-defined and independent of the particular realization of the sequence of shocks and of the initial capital stock. Therefore, the average consumption of an infinitely lived representative agent is independent of the prevailing history of perturbations. Since the shocks are assumed to be ergodic processes, average consumption is equal to the mean of consumption at the random fixed point. A central result of this paper then ensures that the average consumption is a continuous function of the saving rate. A direct consequence of these findings is the existence of a golden rule saving rate yielding maximal average consumption. We also prove a dynamic efficiency result which ensures the importance of the golden rule in the stochastic Solow growth model. Our results are illustrated numerically for Cobb-Douglas and CES production function.

The remainder of the paper is organized as follows. Section 2 introduces the stochastic Solow model and explains the model of the parameter fluctuations. In Section 3 we analyze the long-run behavior of the stochastic Solow model using the concept of a random dynamical system. Section 4 contains the main result of this paper on the existence of a golden rule saving rate. Dynamic efficiency of the golden rule path is proved in Section 5. Two examples are studied in Section 6. 


\section{The Model}

In this paper, we analyze the generalization of the neoclassical Solow (1956) growth model given by

$$
k_{t+1}=h_{s}\left(\theta^{t} \omega, k_{t}\right):=\frac{\left(1-\delta\left(\theta^{t} \omega\right)\right) k_{t}+s \xi\left(\theta^{t} \omega\right) f\left(k_{t}\right)}{1+n\left(\theta^{t} \omega\right)}
$$

The stochastic parameters depreciation rate $\delta\left(\theta^{t} \omega\right)$, population growth rate $n\left(\theta^{t} \omega\right)$, and production shock $\xi\left(\theta^{t} \omega\right)$ are assumed to be ergodic processes. This models exogenously determined stationary fluctuations of the respective parameters. Fluctuations in the population growth rate represent changes in the birth and death rates as well as labor migration. A discussion on exogenous stochastic depreciation rates is provided by Ambler and Paquet (1994). The saving rate $s$ is assumed to be deterministic parameter, fixed for all times. $f$ is a neoclassical production function. At each period of time, the capital intensity $k_{t+1}$ in the next period is a random variable whose state is determined by the realization of the stochastic parameters $\delta\left(\theta^{t} \omega\right), n\left(\theta^{t} \omega\right)$, and $\xi\left(\theta^{t} \omega\right)$, and by the capital intensity $k_{t}$ in the current period.

Throughout the paper we impose the following conditions on the parameters:

(i) $\delta(\omega) \in\left[\delta_{\min }, \delta_{\max }\right] \subset[0,1]$

(ii) $\left.n(\omega) \in\left[n_{\min }, n_{\max }\right] \subset\right]-1, \infty[$

(iii) $\xi(\omega) \in\left[\xi_{\text {min }}, \infty[\subset] 0, \infty[\right.$, and $\mathbb{E} \xi<\infty$

(iv) $\delta_{\max }+n_{\max }>0$

Under these assumptions, the stochastic rate of population growth is in a compact interval and there is a lower bound on the production shock. The production function satisfies an Inada-type condition:

(v) $f: \mathbb{R}_{+} \rightarrow \mathbb{R}_{+}$is increasing, strictly concave, and continuously differentiable. $0 \leq \lim _{k \rightarrow \infty} f^{\prime}(k)<\left(\delta_{\max }+n_{\max }\right) / \xi_{\min }$. If $f(0)=0$, then $f^{\prime}(0)=\infty$. (No condition is needed if $f(0)>0$.)

Using a mathematical description of the stochastic parameters from ergodic theory, we can model stochastic processes as dynamical systems. Let $(\Omega, \mathcal{F}, \mathbb{P})$ denote a probability space, and let $\theta: \Omega \rightarrow \Omega$ be a bi-measurable map (i.e. $\theta$ is measurable and $\theta^{-1}$ exists and is measurable) such that $\theta$ is ergodic with respect to $\mathbb{P}$ (i.e. for all $F \in \mathcal{F}$ such that $\theta F=F, \mathbb{P}(F) \in\{0,1\}$ ). 
Then the family of mappings $\theta^{t}: \Omega \rightarrow \Omega, t \in \mathbb{Z}$ forms a measurable flow, i.e. $\theta^{0}=\operatorname{id}_{\Omega}, \theta^{t}=\theta \circ \ldots \circ \theta$ (t-times) for $t>0$, and $\theta^{-t}=\theta^{-1} \circ \ldots \circ \theta^{-1}$ for $-t<0$. The tupel $\left(\Omega, \mathcal{F}, \mathbb{P},\left(\theta^{t}\right)_{t \in \mathbb{Z})}\right.$ is called an ergodic dynamical system. For each measurable function $g: \Omega \rightarrow \mathbb{R}, g\left(\theta^{t} \omega\right)$ is an ergodic process defined for all times $t \in \mathbb{Z}$. In particular, any real-valued ergodic process has such a representation, cf. Arnold (1998, Appendix A.1). The canonical representation is as follows. Let $\xi_{t}$ be an ergodic process with values in a Borel set $X \subset \mathbb{R}$. Define the sample-path space $\Omega=X^{\mathbb{Z}}$, the Borel $\sigma$-algebra $\mathcal{F}=\mathcal{B}(\Omega)$, and let $\mathbb{P}$ denote the probability measure which is defined by the finite-dimensional distributions of $\xi_{t}$. (Existence follows from Kolmogorov's fundamental theorem.) For instance, if $\xi_{t}$ is an i.i.d. process with distribution $\lambda$ then $\mathbb{P}=\lambda^{\mathbb{Z}}$ is the product measure. The mapping $\theta$ is defined as $\theta^{t} \omega(\cdot):=\omega(t+\cdot)$. The sequence of random variables $\Omega \rightarrow X$, $\omega \mapsto \theta^{t} \omega(0)=\omega(t)$ is the process we started with.

In (1), the stochastic parameters are defined over a common ergodic dynamical system. However, note that these processes may have arbitrary correlation and, in particular, may be independent.

Let us briefly discuss the implications of assumptions (i) $-(\mathrm{v})$ for the deterministic Solow model which is obtained from (1) by choosing the ergodic processes $\delta(\omega) \equiv \delta, n(\omega) \equiv n$, and $\xi(\omega) \equiv \xi$. Then (1) becomes

$$
k_{t+1}=h_{s}\left(k_{t}\right):=\frac{(1-\delta) k_{t}+s \xi f\left(k_{t}\right)}{1+n}
$$

the Solow model with production function $\xi f(k)$.

If $n+\delta>0, s \xi>0$, and $f$ satisfies (v), then (2) has a unique positive fixed point, denoted by $\bar{k}_{s}(\xi, n, \delta)$, which is globally asymptotically stable. This result is obtained as follows: Fix any saving rate $s>0$. Then condition (v) implies $h_{s}(k)>k$ for all sufficiently small $k>0$, and $h_{s}(k)<k$ for all sufficiently large $k>0$. We further have that $h_{s}(k)$ is continuous in $k$, increasing, and strictly concave. From these observations one can straightforwardly conclude existence, uniqueness, and global asymptotic stability of $\bar{k}_{s}(\xi, n, \delta)$. For $s=0$, we define $\bar{k}_{0}(\xi, n, \delta):=0$, which is the unique steady state in this case.

The following result is straightforward from conditions (i)-(v) and the preceding discussion.

Lemma 2.1 For each $0 \leq s \leq 1$ the set

$$
G(s):=\left[\bar{k}_{s}\left(\xi_{\min }, n_{\max }, \delta_{\max }\right), \infty[\right.
$$

is forward invariant, i.e. $h_{s}(\omega, G(s)) \subset G(s)$ for all $\omega . \bar{k}_{s}\left(\xi_{\min }, n_{\max }, \delta_{\max }\right)$ denotes the unique positive steady state of (2) with respective parameters. 
This result ensures that for any initial capital intensity larger than or equal to $\bar{k}_{s}\left(\xi_{\min }, n_{\max }, \delta_{\max }\right)$ the economy will never (i.e. for no feasible sequence of shocks and at no subsequent period of time) experience a capital intensity less than $\bar{k}_{s}\left(\xi_{\min }, n_{\max }, \delta_{\max }\right)$.

We also have to make an assumption on the expected value of the contraction rate of the maps $h_{s}(\omega, k)$ at the left-hand boundary of the forward invariant set $G(s)$. This condition ensures convergence of sample-paths of capital intensities. ( $\mathbb{E}$ denotes the expected value with respect to $\mathbb{P}$.)

(vi) There exists a $c>0$ such that

$$
\sup _{0 \leq s \leq 1} \mathbb{E} \log h_{s}^{\prime}\left(\omega, \bar{k}_{s}\left(\xi_{\min }, n_{\max }, \delta_{\max }\right)\right) \leq-c<0
$$

By the defining equation (1) one has that $h_{s}^{\prime}(\omega, k)=\frac{1-\delta(\omega)+s \xi(\omega) f^{\prime}(k)}{1+n(\omega)}$ for $s>0$, and $h_{0}^{\prime}(\omega, k)=(1-\delta(\omega)) /(1+n(\omega))$ for $s=0$.

Assumption (vi) implies that the law of motion $h$ is a contraction (in the sense that the mean value of the rate of contraction is negative) at the capital intensity $\bar{k}_{s}\left(\xi_{\min }, n_{\max }, \delta_{\max }\right)$. Moreover, the rate of contraction is uniformly bounded away from zero with respect to the saving rate $s$. Since $h$ is concave this implies that $h$ is contracting on average (uniformly in $s$ ) at any larger capital intensity. In the deterministic case (vi) is equivalent to asymptotic stability of the fixed point $\bar{k}_{s}(\xi, n, \delta)$ for each $0 \leq s \leq 1$; but this is obviously satisfied under conditions (i) $-(\mathrm{v})$ according to the above discussion.

\section{The Long-Run Behavior}

In this section we present the main result on the long-run behavior of the stochastic Solow model (1). We need to introduce the notion of a random dynamical system which is the key concept in the further analysis. A random dynamical system consists of two ingredients: (1) an ergodic dynamical system as a model of the perturbation and (2) a dynamical system that is coupled to this ergodic dynamical system. Fix any sample-path of the stochastic parameters, i.e. fix any $\omega \in \Omega$, and let $k$ be an initial value for the capital intensity. Denote by $\varphi_{s}(t, \omega, k)$ the state of the system at time $t$ which is determined by (1). That is, $\varphi_{s}$ is generated by iterations of the random map $k \mapsto h_{s}(\omega, k)$ (denoted by $h_{s}(\omega)$ for short): $\varphi_{s}(t, \omega)=$ $h_{s}\left(\theta^{t-1} \omega\right) \circ \ldots \circ h_{s}(\omega)$ for $t>0$, and $\varphi_{s}(0, \omega)=$ id. In particular, the time-one map $\varphi_{s}(1, \omega)=h_{s}(\omega)$. Since $h_{s}(\omega)$ is invertible, one has $\varphi_{s}(t, \omega)=$ 
$h_{s}\left(\theta^{-t} \omega\right) \circ \ldots \circ h_{s}\left(\theta^{-1} \omega\right)$ for $-t<0$. Further, each map $h_{s}(\omega)$ is continuously differentiable and thus $\varphi_{s}(t, \omega): \mathbb{R}_{+} \rightarrow \mathbb{R}_{+}$is continuously differentiable for all $(t, \omega)$.

The family of maps $\varphi_{s}(t, \omega, k)$ defined by (1) forms a random dynamical system on $\mathbb{R}_{+}$over the ergodic dynamical system $\left(\Omega, \mathcal{F}, \mathbb{P},\left(\theta^{t}\right)_{t \in \mathbb{Z}}\right)$ modeling the perturbation. That is, $\varphi_{s}: \mathbb{Z} \times \Omega \times \mathbb{R}_{+} \rightarrow \mathbb{R}_{+},(t, \omega, k) \mapsto \varphi_{s}(t, \omega, k)$ is a measurable mapping such that $\varphi_{s}(0, \omega)=\operatorname{id}_{\mathbb{R}_{+}}$and $\varphi_{s}(t+u, \omega)=$ $\varphi_{s}\left(t, \theta^{u} \omega\right) \circ \varphi_{s}(u, \omega)$ for all $u, t \in \mathbb{Z}$ and all $\omega \in \Omega$. These properties replace the flow property of a deterministic dynamical system that is generated by the iteration of a map.

The notions 'almost surely' (a.s.) and 'almost all' refer to the measure $\mathbb{P}$ of the ergodic dynamical system which models the exogenous perturbation.

Theorem 3.1 Suppose conditions (i)-(vi) are satisfied. Then the random dynamical system generated by the stochastic Solow model (1) possesses a unique positive random fixed point $k_{s}^{\star}(\omega)$ for each $s>0$, i.e. $\Omega \rightarrow \mathbb{R}_{++}$, $\omega \mapsto k_{s}^{\star}(\omega)$ is a random variable such that a.s.

$$
k_{s}^{\star}(\theta \omega)=h_{s}\left(\omega, k_{s}^{\star}(\omega)\right)
$$

The random fixed point $k_{s}^{\star}(\omega)$ has the following properties.

(i) $k_{s}^{\star}(\omega)$ is globally asymptotically stable, i.e. for all $k>0$

$$
\lim _{t \rightarrow \infty}\left|\varphi_{s}(t, \omega, k)-k_{s}^{\star}\left(\theta^{t} \omega\right)\right|=0 \quad \text { a.s. }
$$

(ii) $k_{s}^{\star}(\omega)$ is measurable with respect to the past $\mathcal{F}^{-}$which is defined as the $\sigma$-algebra $\mathcal{F}^{-}=\sigma\left\{\omega \mapsto \varphi_{s}\left(u, \theta^{-t} \omega\right) \mid 0 \leq t \leq u\right\}$.

(iii) $k_{s}^{\star}(\omega)$ is tempered, i.e. $\lim \sup _{t \rightarrow \infty}\left(\log ^{+} k_{s}^{\star}\left(\theta^{t} \omega\right)\right) / t=0$ a.s. Further, any tempered random variable $y: \Omega \rightarrow \mathbb{R}_{++}$converges to $k_{s}^{\star}(\omega)$ in the sense that $\lim _{t \rightarrow \infty} \varphi_{s}\left(t, \theta^{-t} \omega, y\left(\theta^{-t} \omega\right)\right)=k_{s}^{\star}(\omega)$ a.s.

(iv) If $\mathbb{E} k_{s}^{\star}<\infty$, the empirical law of each sample-path $\varphi_{s}(t, \omega, k)$ with $k>0$ is equal to $k_{s}^{\star} \mathbb{P}$, i.e. $\lim _{T \rightarrow \infty} 1 / T \sum_{t=0}^{T} 1_{B}\left(\varphi_{s}(t, \omega, k)\right)=k_{s}^{\star} \mathbb{P}(B)$ a.s. for each measurable set $B .1_{B}: \mathbb{R}_{+} \rightarrow\{0,1\}$ denotes the indicator map, i.e. $1_{B}(k)=1$ if and only if $k \in B$.

For $s=0, k_{0}^{\star}(\omega) \equiv 0$ is a unique globally asymptotically stable random fixed point, possessing all above properties. 
The proof of Theorem 3.1 can be found in the appendix.

A random fixed point is a random variable that is defined over the ergodic dynamical system which models the exogenous perturbation. For each sample-path of the perturbation, i.e. for each fixed $\omega \in \Omega$, the random fixed point defines a sample-path in the state space via $t \mapsto k_{s}^{\star}\left(\theta^{t} \omega\right)$. Equation (3) says that the random fixed point $k_{s}^{\star}(\omega)$ generates a stationary solution to the stochastic Solow model in the sense that $k_{s}^{\star}\left(\theta^{t+1} \omega\right)=h_{s}\left(\theta^{t} \omega, k_{s}^{\star}\left(\theta^{t} \omega\right)\right)$ for all $t \in \mathbb{Z}$. If the stochastic variables are trivial, i.e. constants, then a random fixed point is simply a fixed point of the corresponding deterministic system. In this case, we reobtain from Theorem 3.1 the results already discussed for the deterministic Solow model.

The stability result (i) says that the evolution of the capital intensity is uniquely determined by the random fixed point. More precisely, for almost any sample-path of the stochastic variables, the sample-path of any capital intensity with positive initial value converges toward the sample-path of the random fixed point, cf. (4). The interpretation of this result is straightforward. Any economic policy that causes a temporary change in the law of motion of the economy (1) and that leads to a different capital intensity cannot have a long-run effect. After abandoning this policy, the capital intensity converges again toward the sample-path of the random fixed point.

Result (ii) is not only of technical interest. It asserts that the state of the random fixed point at each period of time can be constructed from the sole knowledge of the past, i.e. consumers do not have to correctly anticipate future events to determine its state.

Result (iii) is a technical result which is needed in the following. Temperedness of the random fixed point tells us that almost any sample-path $t \mapsto k_{s}^{\star}\left(\theta^{t} \omega\right)$ grows sub-exponentially fast. According to a result due to O'Brien (1982), the only alternative would be that almost any sample-path grows faster than $\exp (a t)$ for any finite $a>0$. See Arnold (1998, Section 4.1.1), for a thorough discussion of this topic. The contraction property, $\lim _{t \rightarrow \infty} \varphi_{s}\left(t, \theta^{-t} \omega, y\left(\theta^{-t} \omega\right)\right)=k_{s}^{\star}(\omega)$ for any tempered positive random variable $y$, provides information on the "attraction strength" of the random fixed point $k_{s}^{\star}(\omega) \cdot \varphi_{s}\left(t, \theta^{-t} \omega, y\left(\theta^{-t} \omega\right)\right)$ is the capital intensity at time zero of an economy with capital intensity $y\left(\theta^{-t} \omega\right)$ at time $-t<0$, i.e. in the past. Therefore, any sequence of economies, indexed by $t$, which has the property that each economy $t$ starts at time $-t$ with a capital intensity not exceeding $y\left(\theta^{-t} \omega\right)$, leads to a sequence of capital intensities at time zero that converges toward $k_{s}^{\star}(\omega)$.

Result (iv) is a statement on the statistical property of each samplepath of capital intensities. If agents average over observed capital inten- 
sities, they obtain a stable feature of its long-run distribution: regardless of the period of time at which the measurement is started, the empirical measure converges to the probability measure $k_{s}^{\star} \mathbb{P}$. If the random variables $\left(\xi\left(\theta^{t} \omega\right), n\left(\theta^{t} \omega\right), \delta\left(\theta^{t} \omega\right)\right), t \in \mathbb{Z}$ are i.i.d. (independent and identically distributed), property (iv) ensures that $k_{s}^{\star} \mathbb{P}$ is a Markov equilibrium. The Markov equilibrium is stable in the sense that it is equal to the empirical measure for almost any sample-path. This renders the corresponding results in Mirman $(1972,1973)$.

The following result is central to ensure existence of a golden rule in the next section. The proof is contained in the appendix.

Proposition 3.2 The random fixed point $k_{s}^{\star}(\omega)$, defined in Theorem 3.1, is continuous and strictly increasing in the saving rate $s \in[0,1]$.

\section{Existence of the Golden Rule}

We are now in a position to study the influence of the saving rate on the consumption in the stochastic Solow model. Analogous to the deterministic case we ask whether there is a saving rate such that average consumption per capita is maximal.

For any sample-path of the stochastic variables, consumption in stochastic Solow model at any period $t$ is given by $(1-s) \xi\left(\theta^{t} \omega\right) f(\varphi(t, \omega, k))$. We know from Theorem 3.1 that the long-run behavior of an economy with a fixed saving rate $s$ is uniquely determined by the sample-path $t \mapsto k_{s}^{\star}\left(\theta^{t} \omega\right)$. Therefore, average consumption will also be determined by the random fixed point. Let us assume $\mathbb{E} \xi f\left(k_{1}^{\star}\right)<\infty$. Then, by Theorem 3.1 (i) and continuity of $f$, the ergodic theorem yields that a.s.

$$
\lim _{T \rightarrow \infty} \frac{1}{T} \sum_{t=0}^{T}(1-s) \xi\left(\theta^{t} \omega\right) f\left(\varphi_{s}(t, \omega, k)\right)=(1-s) \mathbb{E}\left(\xi(\omega) f\left(k_{s}^{\star}(\omega)\right)\right)
$$

for all $k>0$. This implies that the average consumption along almost all sample-paths is well-defined and equal to the expected value of the consumption $c_{s}^{\star}(\omega):=(1-s) \xi(\omega) f\left(k_{s}^{\star}(\omega)\right)$ at the random fixed point $k_{s}^{\star}(\omega)$. Obviously, $c_{1}^{\star}(\omega) \equiv 0$, and $c_{0}^{\star}(\omega) \equiv 0$ by Theorem 3.1. For the deterministic Solow model, $c_{s}^{\star}(\omega)$ is equal to the consumption at the non-trivial steady state.

We are now in a position to state the main result of this paper.

Theorem 4.1 Suppose conditions (i)-(vi) are satisfied. If $\mathbb{E} \xi f\left(k_{1}^{\star}\right)<\infty$, then there exists a golden rule. That is, there is a saving rate $s^{\text {gold }} \in[0,1]$ 
such that average consumption, $\mathbb{E} c_{s}^{\star}=(1-s) \mathbb{E} \xi f\left(k_{s}^{\star}\right)$, is maximal for $s=$ $s^{\text {gold }}$.

Theorem 4.1 extends the well-known result on the existence of a golden rule saving rate for the Solow model to the stochastic case. In the proof, which is relegated to the appendix, it is verified that $\mathbb{E} c_{s}^{\star}$ depends continuously on the saving rate. The applicability of this result is fostered by the next two Corollaries in which sufficient conditions for $\mathbb{E} \xi f\left(k_{1}^{\star}\right)<\infty$ are given that are simple to check for specific parameters.

Corollary 4.2 Suppose conditions (i)-(vi) are satisfied. Then there exists a golden rule, if

(a) $f$ is bounded, or

(b) $\delta_{\min }+n_{\min }>0, \xi(\omega) \in\left[\xi_{\min }, \xi_{\max }\right]$, and $\lim _{k \rightarrow \infty} f^{\prime}(k)<\frac{\delta_{\min }+n_{\min }}{\xi_{\max }}$

If the only stochastic perturbations are i.i.d. production shocks, we obtain the following result.

Corollary 4.3 Suppose conditions (i)-(vi) are satisfied, $\xi$ is an i.i.d. process, and $\delta$ and $n$ are constants. Then the following holds. If $\lim _{k \rightarrow \infty} f^{\prime}(k)<$ $(\delta+n) / \mathbb{E} \xi$, then there exists a golden rule.

The golden rule saving rate has to be determined numerically in general because of the lack of a characterization via first-order conditions. However, for a certain class of production functions, we can prove that the golden rule for the stochastic model coincides with the one for the associated deterministic model. In this case, the golden rule is determined by an analytical expression. The result applies to the case of a Cobb-Douglas technology, see Section 6.

Theorem 4.4 Suppose conditions (i)-(vi) are satisfied, and $\mathbb{E} \xi f\left(k_{1}^{\star}\right)<\infty$. If there exists a continuously differentiable function $g:[0,1] \rightarrow[0,1]$ such that for all $k>0$

$$
s f(g(s) k)=g(s) f(k)
$$

then any golden rule fulfills

$$
\frac{\partial}{\partial s}\left(\frac{1-s}{s} g(s)\right)=0
$$

If (7) has a unique solution, then this solution is the unique golden rule. 
If the production function has the "weighted" homogeneous-of-degreeone property (6), the random fixed point $k_{s}^{\star}(\omega)$ depends on the saving rate in a very particular way: One has that $k_{s}^{\star}(\omega)=g(s) k_{1}^{\star}(\omega)$, see the proof of Theorem 4.4 in the appendix. One also obtains a simple formula for average consumption: $\mathbb{E} c_{s}^{\star}(\omega)=\frac{1-s}{s} g(s) \mathbb{E}\left(\xi(\omega) f\left(k_{1}^{\star}(\omega)\right)\right)$. From this equation the characterization of the golden rule given by $(7)$ is straightforward. The proof also leads to the following

Remark 4.5 (i) Under the assumptions of Theorem 4.4 the golden rule depends only on parameters of the production function.

(ii) The function $g(s)$ in Theorem 4.4 is strictly increasing by Proposition 3.2. Further, $g(0)=0$ and $g(1)=1$.

\section{Dynamic Efficiency}

We derive a dynamic efficiency result for the stochastic Solow model which reveals the importance of the golden rule for that model. It is useful to recall the dynamic efficiency result for the deterministic model first.

In the absence of shocks, consumption is strictly decreasing in all capital stocks which exceed the capital intensity $k_{s \text { gold }}^{\star}$, the fixed point which is assigned to the golden rule saving rate $s^{\text {gold }}$ in the deterministic case. This observation immediately implies that any feasible sample-path of capital intensities $\tilde{k}_{t}$ with $\tilde{k}_{t} \geq k_{s^{\text {gold }}}^{\star}$ and $\tilde{k}_{t}>k_{s^{\text {gold }}}^{\star}$ for at least one $t$ cannot be dynamically efficient: increasing consumption in each period in which $\tilde{k}_{t}>k_{s^{\text {gold }}}^{\star}$ such that the next period's capital stock is not lower than $k_{s^{\text {gold }}}^{\star}$ raises consumption in these periods while it does not harm consumers in all other periods.

In the stochastic case, average consumption, i.e. consumption averaged along sample-paths of the capital intensity, is considered, and the golden rule is defined as the saving rate $s^{\text {gold }}$ for which average consumption is maximal. Note that in the deterministic case average consumption along each sample-path is equal to consumption at the steady state. Since all sample-paths along which consumption only differs at most in finitely many periods yield the same average consumption, our dynamic inefficiency result can only cover the case in which consumption is altered in infinitely many periods. This motivates the following definition.

Definition 5.1 A stochastic process of capital intensities $\tilde{k}(\omega)$ is called dynamically inefficient if average consumption along almost any feasible 
sample-path $t \mapsto \tilde{k}\left(\theta^{t} \omega\right)$ is strictly lower than average consumption associated to the golden rule saving rate, i.e.

$$
\mathbb{E} \tilde{c}(\omega)<\mathbb{E} c_{s^{\text {gold }}}^{\star}(\omega)
$$

where $\tilde{c}(\omega):=(1-\delta(\omega)) \tilde{k}(\omega)+\xi(\omega) f(\tilde{k}(\omega))-(1+n(\omega)) \tilde{k}(\theta \omega)$. A samplepath of capital intensities $\tilde{k}(\omega)$ is called feasible if $\tilde{c}(\omega) \geq 0$ a.s.

By the ergodic theorem, average consumption along sample-paths is equal to the expected value of consumption at the random fixed point for any period of time almost surely. Therefore, average consumption along any dynamically inefficient stochastic process of capital intensities is strictly lower than along almost any sample-path at the golden rule saving rate.

Let us first show existence of stochastic processes with feasible samplepaths along which capital intensities are greater than those associated to the golden rule. We prove that there is a constant $\bar{\varepsilon}>0$ such that for all processes $\varepsilon(\omega)$ with $0 \leq \varepsilon(\omega) \leq \bar{\varepsilon}$ for all $\omega$ and $\mathbb{E} \varepsilon(\omega)>0$, the sample-paths $t \mapsto k_{s^{\text {gold }}}^{\star}\left(\theta^{t} \omega\right)+\varepsilon\left(\theta^{t} \omega\right)$ are feasible. Obviously it suffices to show that there is an $\bar{\varepsilon}>0$ such that for all $\omega$

$$
k_{s^{\text {gold }}}^{\star}(\theta \omega)+\bar{\varepsilon} \leq \frac{(1-\delta(\omega)) k_{s^{\text {gold }}}^{\star}(\omega)+\xi(\omega) f\left(k_{s^{\text {gold }}}^{\star}(\omega)\right)}{1+n(\omega)}
$$

Using (1) and the random fixed point property, this equation is equivalent to

$$
\bar{\varepsilon} \leq \frac{\left(1-s^{\text {gold }}\right) \xi(\omega) f\left(k_{s^{\text {gold }}}^{\star}(\omega)\right)}{1+n(\omega)}
$$

We readily obtain the existence result from this relation, noting that

$0<\frac{\left(1-s^{\text {gold }}\right) \xi_{\min } f\left(\bar{k}_{s^{\text {gold }}}\left(\xi_{\min }, n_{\max }, \delta_{\max }\right)\right)}{1+n_{\max }} \leq \frac{\left(1-s^{\text {gold }}\right) \xi(\omega) f\left(k_{s^{\text {gold }}}^{\star}(\omega)\right)}{1+n(\omega)}$

by conditions (i) $-(\mathrm{v})$.

We can now state the main result of this section, see the appendix for the proof.

Theorem 5.2 Suppose conditions (i)-(vi) are satisfied, $\mathbb{E} \xi f\left(k_{1}^{\star}\right)<\infty$, and

$$
\mathbb{E}\left[\delta(\omega)+n(\omega)-\xi(\omega) f^{\prime}\left(k_{s^{\text {gold }}}^{\star}(\omega)\right)\right] \geq 0 .
$$

Each policy which changes the capital intensity $k_{s^{\text {gold }}}^{\star}(\omega)$ associated to the golden rule by a feasible amount $\varepsilon(\omega)$ with 
(a) $\mathbb{E} \varepsilon(\omega)>0$; and

(b) $\operatorname{Cov}\left(\varepsilon(\omega), \delta(\omega)+n\left(\theta^{-1} \omega\right)-\xi(\omega) f^{\prime}\left(k_{s^{g o l d}}^{\star}(\omega)\right)\right) \geq 0$

leads to a dynamically inefficient stochastic process of capital intensities.

Theorem 5.2 states that any deviation $t \mapsto \varepsilon\left(\theta^{t} \omega\right)$ with $\mathbb{E} \varepsilon>0$ from the sample-path $t \mapsto k_{\text {sgold }}^{\star}\left(\theta^{t} \omega\right)$ is disadvantageous in the sense that it leads to a dynamically inefficient outcome.

Equation (8) requires that average marginal productivity at the random fixed point associated with golden rule should not exceed the mean value of the sum of population growth rate and depreciation. In the deterministic case condition (8) is satisfied because $\delta+n=\xi f^{\prime}\left(k_{s^{g o l d}}^{\star}\right)$ which characterizes the golden rule via the first-order condition.

Condition (a) ensures that the feasible stochastic processes considered lead to a larger average capital intensity than that associated to the golden rule saving rate. As already pointed out above, any sample-path with $\mathbb{E}\left(\tilde{k}(\omega)-k_{\text {sgold }}^{\star}(\omega)\right)=0$ does not change average consumption and, from this point of view, does not represent a policy that is distinguishable from obeying the golden rule. However, the condition $\mathbb{E} \varepsilon(\omega)>0$ does not rule out feasible stochastic processes with lower capital intensities than the golden rule path at infinitely many periods in time. Such sample-paths may well be dynamically inefficient.

Condition (b) restricts the feasible deviations from the golden rule path by requiring a positive correlation between the difference of the sum of depreciation and (previous period's) population growth rate and marginal productivity. (b) is obviously fulfilled for a constant positive deviation from the golden rule path.

\section{Two Examples}

Two examples are analyzed to illustrate our theoretical results. For the standard cases of Cobb-Douglas resp. CES production function, we derive the golden rule saving rate and study numerically the effect of the different stochastic parameters on average consumption. In both cases we consider three stochastic models and, for comparison, the associated deterministic model in which all parameters are set to their respective expected value. The specifications are

(a) Deterministic: $\xi=1, n=0$, and $\delta=1 / 2$

(b) Production shocks: $\xi \in\{3 / 4,5 / 4\}, n=0$, and $\delta=1 / 2$ 
(c) Stochastic population growth: $\xi=1, n \in\{ \pm 7 / 100\}$, and $\delta=1 / 2$

(d) Stochastic depreciation: $\xi=1, n=0$, and $\delta \in\{1 / 3,2 / 3\}$

In each model, the stochastic parameter is assumed to be an ergodic process which follows a two-state irreducible Markov chain with transition probabilities $p_{11}=0.9, p_{12}=0.1, p_{21}=0.1$, and $p_{22}=0.9$. The stationary measure assigns equal probability to both states.

\section{Cobb-Douglas Production Function}

$$
f(k)=A k^{\alpha} \text { with } A>0, \text { and } 0<\alpha<1
$$

It is obvious that assumption (v) follows from assumptions (i)-(iv). A simple sufficient condition for (vi), which allows to check that our results apply in the cases (a)-(d), can be derived as follows. First note that for all $s>0$,

$$
\bar{k}_{s}\left(\xi_{\min }, n_{\max }, \delta_{\max }\right)=\left(\left(\delta_{\max }+n_{\max }\right) /\left(s A \xi_{\min }\right)\right)^{1 /(\alpha-1)}
$$

and therefore $f^{\prime}\left(\bar{k}_{s}\left(\xi_{\min }, n_{\max }, \delta_{\max }\right)\right)=\alpha\left(\delta_{\max }+n_{\max }\right) /\left(s \xi_{\min }\right)$. As a consequence one obtains for each $s>0$

$$
h_{s}^{\prime}\left(\omega, \bar{k}_{s}\left(\xi_{\min }, n_{\max }, \delta_{\max }\right)\right)=\frac{1-\delta(\omega)+\alpha \frac{\xi(\omega)}{\xi_{\min }}\left(\delta_{\max }+n_{\max }\right)}{1+n(\omega)}
$$

Since the right-hand side of the last equation is independent of $s$, and for $s=0, \bar{k}_{0}\left(\xi_{\min }, n_{\max }, \delta_{\max }\right)=0$ and $h_{0}^{\prime}(\omega, 0)=\frac{1-\delta(\omega)}{1+n(\omega)}$, condition (vi) turns out to be equivalent to

$$
\mathbb{E} \log \frac{1-\delta(\omega)+\alpha \frac{\xi(\omega)}{\xi_{\min }}\left(\delta_{\max }+n_{\max }\right)}{1+n(\omega)}<0
$$

Increasing the right-hand side by interchanging $\mathbb{E}$ and log and replacing $n(\omega)$ by $n_{\text {min }}$, we find a sufficient condition for (11):

$$
\alpha<\frac{\xi_{\min }}{\mathbb{E} \xi} \frac{\mathbb{E} \delta(\omega)+n_{\min }}{\delta_{\max }+n_{\max }}
$$

In the deterministic case, i.e. if all ergodic processes are constants, (12) reduces to $\alpha<1$ (which is no additional restriction). It is noteworthy that $\alpha \leq\left[\xi_{\min }\left(\mathbb{E} \delta(\omega)+n_{\min }\right)\right] /\left[\mathbb{E} \xi\left(\delta_{\max }+n_{\max }\right)\right]$ implies (11) (and thus is a sufficient condition for (vi)) if at least one ergodic process is non-degenerated. This follows from the fact that in this case interchanging $\mathbb{E}$ and log strictly increases the left-hand side of (11).

Summarizing, we obtain 
Lemma 6.1 The stochastic Solow model with Cobb-Douglas production function satisfies condition (vi) for all $\alpha$ which fulfill (12).

Let us next determine the golden rule saving rate. For the deterministic Solow model we find by (10) that

$$
c_{s}=(1-s) \xi A\left(\bar{k}_{s}(\xi, n, \delta)\right)^{\alpha}=(1-s) s^{\frac{\alpha}{1-\alpha}} \xi A\left(\frac{\delta+n}{A \xi}\right)^{\frac{\alpha}{\alpha-1}}
$$

Solving $\frac{\partial}{\partial s} c_{s}=0$, we readily obtain the well-known result that $c_{s}$ is maximal for $s=\alpha$. Note that the golden rule $s^{\text {gold }}=\alpha$ is independent of the parameters $\delta, n$, and $\xi$.

The fact that the golden rule depends only on the production function is a hint that Theorem 4.4 might apply, see Remark 4.5. (13) leads to the "guess" $g(s)=s^{\frac{1}{1-\alpha}}$. We need to check first that $s f(g(s) k)=g(s) f(k)$ for all $k>0$, cf. condition (6). This is equivalent to

$$
s A\left(s^{\frac{1}{1-\alpha}} k\right)^{\alpha}=s^{\frac{1}{1-\alpha}} A k^{\alpha}
$$

which is obviously true for all $k>0$. It is straightforward to check that $(\partial / \partial s)(1-s) g(s) / s=0$ has a unique interior solution. Summarizing, we obtain that the golden rules in both deterministic and stochastic case coincide. We may state:

Corollary 6.2 Consider the stochastic Solow model with Cobb-Douglas production function. Suppose conditions (i)-(vi) are satisfied and $\mathbb{E} \xi f\left(k_{1}^{\star}\right)<$ $\infty$. Then the (unique) golden rule saving rate is given by $s^{\text {gold }}=\alpha$, i.e. $\mathbb{E} c_{s}^{\star}$ is maximal for $s=\alpha$.

We now fix the parameters of the Cobb-Douglas production function; let $A=10$ and $\alpha=3 / 4$. It is straightforward to check that for these parameter values conditions (i)-(v) and the sufficient condition (12) for (vi), see Lemma 6.1, are satisfied in each case (a)-(d). Since $\delta_{\min }+n_{\min }>0$ and $0=\lim _{k \rightarrow \infty} f^{\prime}(k)<\left(\delta_{\min }+n_{\min }\right) / \xi_{\max }$, Corollary $4.2(\mathrm{~b})$ ensures that $\mathbb{E} \xi f\left(k_{1}^{\star}\right)<\infty$. Summarizing, we have found that a non-trivial random fixed point exists for all positive saving rates, Theorem 3.1, that $\mathbb{E} c_{s}^{\star}(\omega)<\infty$ for all $s$, and that $s^{\text {gold }}=\alpha$, Corollary 4.2.

Table 1 lists the average consumption at the golden rule saving rate in all four cases. The approximation of average consumption per capita $\mathbb{E} c_{s}^{\star}$ with saving rate $s=\alpha=0.75$ has been carried out as follows. First, a samplepath of the length 1,000 is calculated to approximate the random fixed point 
$k_{s}^{\star}\left(\theta^{t} \omega\right)$. Second, a sample-path of the length $1,000,000$ is calculated and average consumption is calculated according to the convergence result (5). The numerical procedure has been implemented in $C$, Schenk-Hoppé (2001).

\begin{tabular}{lccc} 
case & golden rule & av. cons. & av. cons. relative to (a) \\
\hline (a) & 0.75 & $8,437.5$ & $100.0 \%$ \\
(b) & 0.75 & $9,404.0$ & $111.5 \%$ \\
(c) & 0.75 & $8,883.4$ & $105.3 \%$ \\
(d) & 0.75 & $10,203.6$ & $120.9 \%$
\end{tabular}

Table 1: Golden rule saving rate, numerically approximated average consumption per capita at the golden rule, and average consumption per capita relative to the deterministic case (a).

Table 1 documents that average consumption at the golden rule is higher in all stochastic cases (b)-(d) than in the deterministic case (a). If production shocks occur, case (b), the average consumption is more than $11 \%$ higher than in the deterministic case (a). Fluctuations of the depreciation rate, case (d), increase average consumption by more than $20 \%$. A stochastic population growth rate, case (c), leads to a $5.3 \%$ increase of average consumption.

Since the golden rule is the same in all four cases, the gain in average consumption has to be due to the non-linearity of the Solow model or, in case (c), to slower population growth. In case (c), the average population growth rate is $0.9975 \approx \exp (0.5(\log (0.93)+\log (1.07)))<1$. Thus the population size decreases in case (c) whereas it is constant in all other cases. However, this has only a minor effect on the increase of average consumption, because the difference in the population growth rates is approximately $0.25 \%$ and, thus, accounts only for less than $5 \%$ of the increase in average consumption. Therefore the increase in consumption is caused by the non-linearity of the law of motion which is due to the strict concavity of the production function. It is important to point out that for the same perturbation with transition probability $p_{i j}$ replaced by by $1-p_{i j}, i, j=1,2$, i.e. for a fast fluctuating process, average consumption in the stochastic cases is lower than in the deterministic case.

Summarizing, we may conclude that our numerical results provide evidence on the importance to take into account stochastic parameter fluctuations. Replacing a stochastic parameter by its expected value would 
lead to erroneous data on average consumption. Table 1 illustrates that a non-neglectable error would be made.

Finally, we briefly discuss the applicability of the results on dynamic efficiency given in Section 5. The task is to find conditions under which the crucial assumption of Theorem 5.2, (8), is fulfilled. This assumption requires $\mathbb{E}\left[\delta(\omega)+n(\omega)-\xi(\omega) f^{\prime}\left(k_{s^{\text {gold }}}^{\star}(\omega)\right)\right] \geq 0$. We have to resort to numerical simulations to check this condition, Schenk-Hoppé (2001). Our numerical results indicate that condition (8) is satisfied in the following cases. Deterministic case (a): for all $0<\alpha<1$; production shocks (b): for all $\alpha \leq 0.4$; and stochastic population growth (c): for all $0<\alpha<1$. In case (d), stochastic depreciation, the condition is not fulfilled for any $\alpha$.

\section{CES Production Function}

$$
f(k)=\left(1-A+A k^{\alpha}\right)^{1 / \alpha} \text { with } 0 \neq \alpha<1, \text { and } 0<A<1
$$

We first derive a sufficient condition for the indispensable assumption (vi) which ensures applicability of our results. Elementary calculations show that $\bar{k}_{s}\left(\xi_{\min }, n_{\max }, \delta_{\max }\right)=(1-A)^{1 / \alpha}\left[\left(\left(\delta_{\max }+n_{\max }\right) /\left(s \xi_{\min }\right)\right)^{\alpha}-A\right]^{-1 / \alpha}$ and hence $f^{\prime}\left(\bar{k}_{s}\left(\xi_{\text {min }}, n_{\text {max }}, \delta_{\text {max }}\right)\right)=A\left(\left(\delta_{\max }+n_{\text {max }}\right) /\left(s \xi_{\text {min }}\right)\right)^{1-\alpha}$. From these expressions we find that $h_{s}^{\prime}\left(\omega, \bar{k}_{s}\left(\xi_{\min }, n_{\max }, \delta_{\max }\right)\right)=(1-\delta(\omega)+$ $\left.s^{\alpha} \xi(\omega) A\left(\left(\delta_{\max }+n_{\max }\right) / \xi_{\min }\right)^{1-\alpha}\right) /(1+n(\omega))$ for all $s>0$. Therefore, condition (vi) is fulfilled if

$$
\mathbb{E} \log \frac{1-\delta(\omega)+\xi(\omega) A\left(\frac{\delta_{\max }+n_{\max }}{\xi_{\min }}\right)^{1-\alpha}}{1+n(\omega)}<0
$$

Analogous to the Cobb-Douglas case, we get the sufficient condition for (vi)

$$
A<\frac{\xi_{\min }^{1-\alpha}}{\mathbb{E} \xi} \frac{\mathbb{E} \delta+n_{\min }}{\left(\delta_{\max }+n_{\max }\right)^{1-\alpha}}
$$

The Inada condition $(\mathrm{v}) \lim _{k \rightarrow \infty} f^{\prime}(k)=\lim _{k \rightarrow \infty} A\left((1-A) k^{-\alpha}+A\right)^{(1-\alpha) / \alpha}$ $=A^{1 / \alpha}$ is satisfied if $A<\left(\left(\delta_{\max }+n_{\max }\right) / \xi_{\min }\right)^{\alpha}$. No condition is needed at the capital intensity zero because $f(0)=(1-A)^{1 / \alpha}>0$.

We now fix the parameters of the CES production function: $A=1 / 2$ and $\alpha=1 / 2$. Analogous to the Cobb-Douglas case one can check that for these parameter values conditions (i) $-(\mathrm{v})$ and the sufficient condition (15) for (vi) are satisfied in the cases (a)-(d). Further it holds $\mathbb{E} \xi f\left(k_{1}^{\star}\right)<\infty$. From this we may conclude that a non-trivial random fixed point exists for 
all positive saving rates, that $\mathbb{E} c_{s}^{\star}(\omega)<\infty$ for all $s$, and that a golden rule exists.

Applying the same approximation procedure as in the preceding case, we obtain the average consumption per capita $\mathbb{E} c_{s}^{\star}$ for each fixed saving rate $s \in[0,1]$ in the cases (a)-(d). The results are collected in Table 2.

\begin{tabular}{lccc} 
case & golden rule & av. cons. & av. cons. relative to (a) \\
\hline (a) & 0.5 & 0.5 & $100.0 \%$ \\
(b) & 0.53 & 0.528 & $105.6 \%$ \\
(c) & 0.52 & 0.51 & $102.0 \%$ \\
(d) & 0.56 & 0.55 & $110.0 \%$
\end{tabular}

Table 2: Stochastic Solow model with CES production function. Numerically approximated golden rule saving rate, average consumption per capita in absolute values, and average consumption per capita relative to the deterministic case (a).

Average consumption is higher in the stochastic cases than in the deterministic case. However, while the ranking of the cases according to maximal average consumption is as in the Cobb-Douglas case, the relative gain is only of about a half of that in the Cobb-Douglas case. The main difference in the CES production function is that the golden rule saving rate depends on the particular case.

The observations made in our case study for the CES production function can be summarized as follows. The stochastic fluctuation of any parameter of the Solow growth model (1) increases average consumption for any saving rate. The golden rule saving rate is higher as well.

Let us close this section with a short remark on dynamic efficiency in the cases (a)-(d). Numerical results indicate that condition (8) of Theorem 5.2 is satisfied in all cases (b)-(d). In the CES example we therefore derive the strongest dynamic efficiency result which is possible by Theorem 5.2.

\section{A Appendix}

Proof of Theorem 3.1. Assertions (i), (ii), and (iv) correspond to Theorem 3 of Schenk-Hoppé and Schmalfuss (in press). Assertion (iii) is immediate from Theorem 2.2 of Schmalfuss (1996). Only the case $s=0$ is not 
covered in these references. However, one has

$$
\varphi_{0}(t+1, \omega, k)=\frac{1-\delta\left(\theta^{t} \omega\right)}{1+n\left(\theta^{t} \omega\right)} \varphi_{0}(t, \omega, k)=\prod_{u=0}^{t} \frac{1-\delta\left(\theta^{u} \omega\right)}{1+n\left(\theta^{u} \omega\right)} k
$$

By condition (vi), this yields assertions (i)-(iv). Observe that $k_{0}^{\star}(\omega) \equiv 0$.

In Schenk-Hoppé and Schmalfuss (in press) the authors apply a Banach fixed point theorem for random maps to prove existence of a random fixed point with the specified properties. This generalization of the well-known Banach theorem for random dynamical systems is due to Schmalfuss (1996, 1998). The main idea of this theorem is to consider the space of all tempered random variables with values in some set $G(\omega)$ which is forward invariant in the sense that $\varphi(t, \omega, G(\omega)) \subset G\left(\theta^{t} \omega\right)$ for all $t \geq 0$, cf. Lemma 2.1. The operator $x \mapsto\left(\lim _{t \rightarrow \infty} \varphi\left(t, \theta^{-t} \omega, x\left(\theta^{-t} \omega\right)\right)\right)_{\omega \in \Omega}$ is a global contraction on that space under a condition that ensures contraction on the average (condition (vi) in the present paper is a particular version of this condition).

Proof of Proposition 3.2. We first prove that $s \mapsto k_{s}^{\star}(\omega)$ is a strictly increasing function. Since $f$ is increasing and $f(k)>0$ for all $k>0$, one has that for all $s<s^{\prime}, k \leq k^{\prime}$

$$
\begin{aligned}
h_{s^{\prime}}\left(\omega, k^{\prime}\right)-h_{s}(\omega, k) & =\frac{(1-\delta(\omega))\left(k^{\prime}-k\right)+\xi(\omega)\left(s^{\prime} f\left(k^{\prime}\right)-s f(k)\right)}{1+n(\omega)} \\
& \geq \frac{\left(s^{\prime}-s\right) \xi(\omega)}{1+n(\omega)} f(k) \geq \frac{\left(s^{\prime}-s\right) \xi_{\text {min }}}{1+n_{\max }} f(k) .
\end{aligned}
$$

Fix any $0<s<s^{\prime}$. Then the preceding estimate ensures existence of a $c\left(s, s^{\prime}\right)>0$ (which is independent of $k$ and $\omega$ ) such that $h_{s^{\prime}}\left(\omega, k^{\prime}\right)-h_{s}(\omega, k) \geq$ $c\left(s, s^{\prime}\right)$ for all $k \in G(s)$ and $k^{\prime} \geq k$, see Lemma 2.1 for the definition of $G(s)$.

Using that $\varphi_{s}\left(t, \theta^{-t} \omega, k\right)=h_{s}\left(\theta^{-1} \omega\right) \circ \ldots \circ h_{s}\left(\theta^{-t} \omega, k\right)$, one finds $\varphi_{s^{\prime}}\left(t, \theta^{-t} \omega, k\right)-\varphi_{s}\left(t, \theta^{-t} \omega, k\right) \geq c\left(s, s^{\prime}\right)$ for all $t \geq 0$. By the contraction property of $k_{s}^{\star}(\omega)$ stated in Theorem 3.1, one finally obtains for $s<s^{\prime}$ and arbitrarily fixed $k \in G(s)$

$$
k_{s}^{\star}(\omega)=\lim _{t \rightarrow \infty} \varphi_{s}\left(t, \theta^{-t} \omega, k\right)<\lim _{t \rightarrow \infty} \varphi_{s^{\prime}}\left(t, \theta^{-t} \omega, k\right)=k_{s^{\prime}}^{\star}(\omega)
$$

which yields that $k_{s}^{\star}(\omega)$ is strictly increasing in $s$.

We next prove continuity of $s \mapsto k_{s}^{\star}(\omega)$. Fix any $\omega$ and any $k>0$. $k_{s}^{\star}(\omega)=\lim _{t \rightarrow \infty} \varphi_{s}\left(t, \theta^{-t} \omega, k\right)$ for all $s \in[0,1]$ by Theorem 3.1. Since $\varphi_{s}\left(t, \theta^{-t} \omega, k\right)$ is continuous in $s$ for each fixed $t$, it suffices to show that 
there exists a $k>0$ such that this convergence is uniformly in $s \in[0,1]$. Letting $\bar{k}:=\bar{k}_{1}\left(\xi_{\min }, n_{\max }, \delta_{\max }\right)$, we find

$$
\begin{array}{r}
\sup _{s \in[0,1]}\left|\varphi_{s}\left(t, \theta^{-t} \omega, \bar{k}\right)-k_{s}^{\star}(\omega)\right|=\sup _{s \in[0,1]}\left|\varphi_{s}\left(t, \theta^{-t} \omega, \bar{k}\right)-\varphi_{s}\left(t, \theta^{-t} \omega, k_{s}^{\star}\left(\theta^{-t} \omega\right)\right)\right| \\
\leq \sup _{s \in[0,1]}\left|k_{s}^{\star}\left(\theta^{-t} \omega\right)-\bar{k}\right| \prod_{u=1}^{t} h_{s}^{\prime}\left(\theta^{-u} \omega, \bar{k}_{s}\left(\xi_{\text {min }}, n_{\text {max }}, \delta_{\text {max }}\right)\right)
\end{array}
$$

where we have used that $\min \left\{\bar{k}, k_{s}^{\star}(\omega)\right\} \geq \bar{k}_{s}\left(\xi_{\min }, n_{\max }, \delta_{\max }\right)$ and $h_{s}^{\prime}(\omega, k)$ is decreasing in $k$.

Assumption (vi) implies that for any $\varepsilon>0$ there exists a $T(\varepsilon)$ such that

$$
\prod_{u=1}^{t} h_{s}^{\prime}\left(\theta^{-u} \omega, \bar{k}_{s}\left(\xi_{\min }, n_{\max }, \delta_{\max }\right)\right) \leq \exp (-(c-\varepsilon) t)
$$

for all $t \geq T(\varepsilon)$, where the exponential rate is independent of $s$. Hence for all $\varepsilon<c$ and $t \geq T(\varepsilon)$

$$
\begin{aligned}
\sup _{s \in[0,1]}\left|\varphi_{s}\left(t, \theta^{-t} \omega, \bar{k}\right)-k_{s}^{\star}(\omega)\right| & \leq \sup _{s \in[0,1]}\left(k_{s}^{\star}\left(\theta^{-t} \omega\right)+\bar{k}\right) \exp (-(c-\varepsilon) t) \\
& =\left(k_{1}^{\star}\left(\theta^{-t} \omega\right)+\bar{k}\right) \exp (-(c-\varepsilon) t)
\end{aligned}
$$

where the last equality follows from $k_{s}^{\star}(\omega)$ being strictly increasing.

Finally, temperedness of $k_{1}^{\star}(\omega)$, i.e. $\limsup _{t \rightarrow \infty} k_{1}^{\star}\left(\theta^{-t} \omega\right) \exp (-\delta t)=0$ for all $\delta>0$, yields that the convergence $k_{s}^{\star}(\omega)=\lim _{t \rightarrow \infty} \varphi_{s}\left(t, \theta^{-t} \omega, k\right)$ is uniform in $s \in[0,1]$. This finishes the proof.

Proof of Theorem 4.1. We prove that $s \mapsto \mathbb{E} c_{s}^{\star}(\omega)$ is a continuous function, this yields the assertion. By Proposition 3.2 and continuity of $f$, the map $s \mapsto c_{s}^{\star}(\omega)=(1-s) \xi(\omega) f\left(k_{s}^{\star}(\omega)\right)$ is continuous. Proposition 3.2 further implies $c_{s}^{\star}(\omega) \leq \xi(\omega) f\left(k_{1}^{\star}(\omega)\right)$ for all $s$. The condition $\mathbb{E} \xi f\left(k_{1}^{\star}\right)<\infty$ ensures that $\mathbb{E} c_{s}^{\star}(\omega)$ exists and is uniformly bounded in $s$. Thus the dominated convergence theorem applies and we are done.

Proof of Corollary 4.2. Theorem 4.1 yields the result, if we can show $\mathbb{E} \xi f\left(k_{1}^{\star}\right)<\infty$ under the respective assumption. (a) is immediate from assumption (iii) which gives integrability of $\xi(\omega)$.

Let us turn to (b). Our assumption ensures existence of a non-trivial fixed point $\bar{k}_{1}\left(\delta_{\min }, n_{\min }, \xi_{\max }\right)$ of the deterministic model (2) with respective parameters. Further one has $h_{1}(\omega, k) \leq\left(\left(1-\delta_{\min }\right) k+\xi_{\max } f(k)\right) /(1+$ $\left.n_{\min }\right)$. Therefore $k_{1}^{\star}(\omega) \leq \bar{k}_{1}\left(\xi_{\max }, n_{\min }, \delta_{\min }\right)$ is uniformly bounded. This yields the result. 
Proof of Corollary 4.3. By Theorem 4.1 it suffices to show that $\mathbb{E} \xi f\left(k_{1}^{\star}\right)<$ $\infty$. Taking expected values on both sides of $(1)$, one obtains $(\delta+n) \mathbb{E} k_{1}^{\star}=$ $\mathbb{E} \xi f\left(k_{1}^{\star}\right)$. Observe that $\delta>0$, because for constant $\delta$ and $n$ assumption (vi) yields $1-\delta+\xi_{\text {min }} f^{\prime}\left(\bar{k}_{1}\right) \leq \exp \left(\mathbb{E} \log \left(1-\delta+\xi(\omega) f^{\prime}(\bar{k})\right)\right)<1+n$ and assumption (iii) gives $\xi_{\min } f^{\prime}\left(\bar{k}_{1}\right)>0$, where $\bar{k}:=\bar{k}_{1}\left(\xi_{\min }, n_{\max }, \delta_{\max }\right)$. It therefore remains to prove $\mathbb{E} k_{1}^{\star}<\infty$. It is

$$
h_{1}(\omega, k) \leq \frac{1-\delta+\xi(\omega) f^{\prime}(y)}{1+n} k+\frac{\xi(\omega) f(y)}{1+n}
$$

because concavity of $f$ implies $f(k) \leq f(y)+f^{\prime}(y) k$ for all $k$ with an arbitrarily fixed $y>0$. Consider the associated affine random difference equation

$$
x_{t+1}=a\left(\theta^{t} \omega\right) x_{t}+b\left(\theta^{t} \omega\right)
$$

with

$$
a(\omega):=\frac{1-\delta+\xi(\omega) f^{\prime}(y)}{1+n} \quad \text { and } \quad b(\omega):=\frac{\xi(\omega) f(y)}{1+n}
$$

where $y \geq \bar{k}_{1}\left(\xi_{\min }, n_{\max }, \delta_{\max }\right)$ is arbitrarily fixed. The random dynamical system generated by (17) possesses the unique globally attracting random fixed point

$$
x^{\star}(\omega):=b\left(\theta^{-1} \omega\right)+\sum_{i=1}^{\infty} b\left(\theta^{-(i+1)} \omega\right) \prod_{j=1}^{i} a\left(\theta^{-j} \omega\right)
$$

which is an upper bound for the random fixed point of the stochastic Solow model, i.e. $k_{1}^{\star}(\omega) \leq x^{\star}(\omega)$ for all $\omega$, see Schenk-Hoppé and Schmalfuss (in press), Proof of Theorem 3 part (d).

We will show that $\mathbb{E} x^{\star}(\omega)<\infty$ which yields the assertion. By the dominated convergence theorem, it suffices to show that for the sequence of random variables $x_{N}(\omega):=b\left(\theta^{-1} \omega\right)+\sum_{i=1}^{N} b\left(\theta^{-(i+1)} \omega\right) \prod_{j=1}^{i} a\left(\theta^{-j} \omega\right)$ the expected value $\mathbb{E} x_{N}$ is uniformly bounded. One has

$$
\begin{aligned}
\mathbb{E} x_{N} & =\mathbb{E} b+\sum_{i=1}^{N} \mathbb{E}\left[b\left(\theta^{-(i+1)} \omega\right) \prod_{j=1}^{i} a\left(\theta^{-j} \omega\right)\right] \leq \mathbb{E} b+\frac{f(y)}{f^{\prime}(y)} \sum_{i=1}^{N} \mathbb{E} \prod_{j=1}^{i+1} a\left(\theta^{-j} \omega\right) \\
& =\mathbb{E} b+\frac{f(y)}{f^{\prime}(y)} \sum_{i=1}^{N}(\mathbb{E} a)^{i+1}=\mathbb{E} b+\frac{f(y)}{f^{\prime}(y)}(\mathbb{E} a)^{2} \frac{1-(\mathbb{E} a)^{N}}{1-\mathbb{E} a}
\end{aligned}
$$

where we have used that $a\left(\theta^{-i} \omega\right)$ and $a\left(\theta^{-j} \omega\right)$ are independent for all $i \neq j$ because $\xi$ is an i.i.d. process by assumption. 
By the Inada condition $\lim _{k \rightarrow \infty} f^{\prime}(k)<(n+\delta) / \mathbb{E} \xi$ there exists a $y \geq \bar{k}$ such that $\mathbb{E} a=\left(1-\delta+\mathbb{E} \xi f^{\prime}(y)\right) /(1+n)<1$. Hence for all sufficiently large $y$ we have

$$
\mathbb{E} x_{N} \leq \mathbb{E} b+\frac{f(y)}{f^{\prime}(y)} \frac{(\mathbb{E} a)^{2}}{1-\mathbb{E} a}
$$

for all $N$, which asserts that $\mathbb{E} x^{\star}<\infty$. This in turn yields $\mathbb{E} k_{1}^{\star}<\infty$ and finishes the proof.

Proof of Theorem 4.4. Theorems 3.1 and 4.1 ensure existence of a unique non-trivial random fixed point $k_{s}^{\star}(\omega)$ and finiteness of $\mathbb{E} c_{s}^{\star}(\omega)=$ $(1-s) \mathbb{E} \xi(\omega) f\left(k_{s}^{\star}(\omega)\right)$ for all $s \in[0,1]$.

We first show that $k_{s}^{\star}(\omega)=g(s) k_{1}^{\star}(\omega) . k_{s}^{\star}(\omega)$ is the unique random fixed point solving (1). Replacing $k_{s}^{\star}(\omega)$ by $g(s) k_{1}^{\star}(\omega)$ in (1), we observe that

$$
\begin{aligned}
g(s) k_{1}^{\star}(\theta \omega) & =\frac{(1-\delta(\omega)) g(s) k_{1}^{\star}(\omega)+s \xi(\omega) f\left(g(s) k_{1}^{\star}(\omega)\right)}{1+n(\omega)} \\
& =g(s) \frac{(1-\delta(\omega)) k_{1}^{\star}(\omega)+\xi(\omega) f\left(k_{1}^{\star}(\omega)\right)}{1+n(\omega)}
\end{aligned}
$$

by assumption (6). Dividing both sides by $g(s)$ gives an equation for $k_{1}^{\star}(\omega)$ which is fulfilled by Theorem 3.1. Hence $k_{s}^{\star}(\omega)=g(s) k_{1}^{\star}(\omega)$ by uniqueness of the solution.

This result implies that average consumption is given by

$$
\mathbb{E} c_{s}^{\star}(\omega)=(1-s) \mathbb{E}\left(\xi(\omega) f\left(g(s) k_{1}^{\star}(\omega)\right)\right)=\frac{1-s}{s} g(s) \mathbb{E}\left(\xi(\omega) f\left(k_{1}^{\star}(\omega)\right)\right)
$$

Since $\mathbb{E} c_{s}^{\star}$ is continuously differentiable in $s$, any maximizer is an interior point, and $\frac{\partial}{\partial s} \mathbb{E} c_{s}^{\star}=0$ if and only if (7) holds, we obtain that (7) is a necessary condition for the golden rule saving rate. If the solution to (7) is unique then (7) is also a sufficient condition.

Proof of Theorem 5.2. We have to show that $\mathbb{E}\left(c_{s^{\text {gold }}}^{\star}(\omega)-c(\omega)\right)>0$ where $c(\omega):=(1-\delta(\omega))\left(k_{\text {sold }}^{\star}(\omega)+\varepsilon(\omega)\right)+\xi(\omega) f\left(k_{s^{\text {gold }}}^{\star}(\omega)+\varepsilon(\omega)\right)-(1+$ $n(\omega))\left(k_{\text {sold }}^{\star}(\theta \omega)+\varepsilon(\theta \omega)\right)$.

It is straightforward to check that $\mathbb{E}\left(c_{s^{\text {gold }}}^{\star}(\omega)-c(\omega)\right)=$ $\mathbb{E}\left[\delta(\omega) \varepsilon(\omega)+n(\omega) \varepsilon(\theta \omega)-\xi(\omega)\left(f\left(k_{s^{\text {gold }}}^{\star}(\omega)+\varepsilon(\omega)\right)-f\left(k_{s^{\text {gold }}}^{\star}(\omega)\right)\right)\right]$.

By condition (vi), $f(y)-f(x) \leq f^{\prime}(x)(y-x)$ with strict inequality if $y \neq x$. The condition $\mathbb{E} \varepsilon>0$ implies that $\varepsilon(\omega)>0$ with positive probability. We therefore obtain, recalling that $\xi(\omega)>0$ for all $\omega$ by condition (iii),

$$
\mathbb{E}\left(c_{s^{g o l d}}^{\star}(\omega)-c(\omega)\right)>\mathbb{E}\left[\delta(\omega) \varepsilon(\omega)+n(\omega) \varepsilon(\theta \omega)-\xi(\omega) f^{\prime}\left(k_{s^{g o l d}}^{\star}(\omega)\right) \varepsilon(\omega)\right]
$$


Finally, rewriting the right-hand side of this expression and employing assumption (b) yields

$$
\begin{aligned}
\mathbb{E}\left(c_{s^{\text {gold }}}^{\star}(\omega)-c(\omega)\right) & >\mathbb{E}\left[\left(\delta(\omega)+n\left(\theta^{-1} \omega\right)-\xi(\omega) f^{\prime}\left(k_{s^{\text {gold }}}^{\star}(\omega)\right)\right) \varepsilon(\omega)\right] \\
\geq & \mathbb{E}\left[\left(\delta(\omega)+n\left(\theta^{-1} \omega\right)-\xi(\omega) f^{\prime}\left(k_{s^{\text {gold }}}^{\star}(\omega)\right)\right)\right] \mathbb{E} \varepsilon(\omega) \geq 0
\end{aligned}
$$

where the last step uses assumption (8). We are done.

\section{References}

Ambler, S. \& A. Paquet (1994) Stochastic depreciation and the business cycle. International Economic Review 35, 101-116.

Arnold, L. (1998) Random Dynamical Systems. Berlin: Springer-Verlag.

Barro, R.J. \& X. Sala-i-Martin (1995) Economic Growth. New York: McGraw-Hill.

Mirman, L.J. (1972) On the existence of steady state measures for one sector growth models with uncertain technology. International Economic Review 13, 271-286.

Mirman, L.J. (1973) The steady state behavior of a class of one sector growth models with uncertain technology. Journal of Economic Theory 6, 219-242.

O'Brien, G.L. (1982) The occurrence of large values in stationary sequences. Probability and Related Fields 61, 347-353.

Phelps, E.S. (1996) Golden Rules of Economic Growth. New York: Norton.

Schenk-Hoppé, K.R. (2001) Software for the stochastic Solow model. Web page http://www.iew.unizh.ch/home/klaus/numerics.

Schenk-Hoppé, K.R. \& B. Schmalfuss (in press) Random fixed points in a stochastic Solow growth model. Journal of Mathematical Economics.

Schmalfuss, B. (1996) A random fixed point theorem based on Lyapunov exponents. Random \& Computational Dynamics 4, 257-268.

Schmalfuss, B. (1998) A random fixed point theorem and the random graph transformation. Journal of Mathematical Analysis and Applications $225,91-113$.

Solow, R.M. (1956) A contribution to the theory of economic growth. Quarterly Journal of Economics 70, 65-94. 


\section{Working Papers of the Institute for Empirical Research in Economics}

No.

1. Rudolf Winter-Ebmer and Josef Zweimüller: Firm Size Wage Differentials in Switzerland: Evidence from Job Changers, February 1999

2. Bruno S. Frey and Marcel Kucher: History as Reflected in Capital Markets: The Case of World War II, February 1999

3. Josef Falkinger, Ernst Fehr, Simon Gächter and Rudolf Winter-Ebmer: A Simple Mechanism for the Efficient Provision of Public Goods - Experimental Evidence, February 1999

4. Ernst Fehr and Klaus M. Schmidt: A Theory of Fairness, Competition and Cooperation, April 1999

5. Markus Knell: Social Comparisons, Inequality, and Growth, April 1999

6. Armin Falk and Urs Fischbacher: A Theory of Reciprocity, July 2000

7. Bruno S. Frey and Lorenz Goette: Does Pay Motivate Volunteers?, May 1999

8. Rudolf Winter-Ebmer and Josef Zweimüller: Intra-firm Wage Dispersion and Firm Performance, May 1999

9. Josef Zweimüller: Schumpeterian Entrepreneurs Meet Engel's Law: The Impact of Inequality on InnovationDriven Growth, May 1999

10. Ernst Fehr and Simon Gächter: Cooperation and Punishment in Public Goods Experiments, June 1999

11. Rudolf Winter-Ebmer and Josef Zweimüller: Do Immigrants Displace Young Native Workers: The Austrian Experience, June 1999

12. Ernst Fehr and Jean-Robert Tyran: Does Money Illusion Matter?, June 1999

13. Stefan Felder and Reto Schleiniger: Environmental Tax Reform: Efficiency and Political Feasibility, July 1999

14. Bruno S. Frey: Art Fakes - What Fakes?, An Economic View, July 1999

15. Bruno S. Frey and Alois Stutzer: Happiness, Economy and Institutions, July 1999

16. Urs Fischbacher, Simon Gächter and Ernst Fehr: Are People Conditionally Cooperative? Evidence from a Public Goods Experiment, July 2000

17. Armin Falk, Ernst Fehr and Urs Fischbacher: On the Nature of Fair Behavior, August 1999

18. Vital Anderhub, Simon Gächter and Manfred Königstein: Efficient Contracting and Fair Play in a Simple Principal-Agent Experiment, September 2000

19. Simon Gächter and Armin Falk: Reputation or Reciprocity?, September 1999

20. Ernst Fehr and Klaus M. Schmidt: Fairness, Incentives, and Contractual Choices, September 1999

21. Urs Fischbacher: z-Tree - Experimenter's Manual, September 1999

22. Bruno S. Frey and Alois Stutzer: Maximising Happiness?, October 1999

23. Alois Stutzer: Demokratieindizes für die Kantone der Schweiz, October 1999

24. Bruno S. Frey: Was bewirkt die Volkswirtschaftslehre?, October 1999

25. Bruno S. Frey, Marcel Kucher and Alois Stutzer: Outcome, Process \& Power in Direct Democracy, November 1999

26. Bruno S. Frey and Reto Jegen: Motivation Crowding Theory: A Survey of Empirical Evidence, November 1999

27. Margit Osterloh and Bruno S. Frey: Motivation, Knowledge Transfer, and Organizational Forms, November 1999

28. Bruno S. Frey and Marcel Kucher: Managerial Power and Compensation, December 1999

29. Reto Schleiniger: Ecological Tax Reform with Exemptions for the Export Sector in a two Sector two Factor Model, December 1999

30. Jens-Ulrich Peter and Klaus Reiner Schenk-Hoppé: Business Cycle Phenomena in Overlapping Generations Economies with Stochastic Production, December 1999

31. Josef Zweimüller: Inequality, Redistribution, and Economic Growth, January 2000

32. Marc Oliver Bettzüge and Thorsten Hens: Financial Innovation, Communication and the Theory of the Firm, January 2000

33. Klaus Reiner Schenk-Hoppé: Is there a Golden Rule for the Stochastic Solow Growth Model? (Revised Version) January 2001

The Working Papers of the Institute for Empirical Research in Economics can be downloaded in PDF-format from http://www.unizh.ch/iew/wp/ 